\title{
Evaluation of personal protection afforded by repellent-treated sandals against mosquito bites in south-eastern Tanzania
}

\author{
Onyango P. Sangoro ${ }^{1,2^{*}}$, Tegemeo Gavana ${ }^{1}$, Marceline Finda ${ }^{1,5}$, Winfrida Mponzi ${ }^{1}$, Emmanuel Hape ${ }^{1}$, \\ Alex Limwagu ${ }^{1}$, Nicodem J. Govella1,3,6, Prosper Chaki ${ }^{1,4}$ and Fredros O. Okumu ${ }^{1,3,5,6}$
}

\begin{abstract}
Background: Outdoor and early evening mosquito biting needs to be addressed if malaria elimination is to be achieved. While indoor-targeted interventions, such as insecticide-treated nets and indoor residual spraying, remain essential, complementary approaches that tackle persisting outdoor transmission are urgently required to maximize the impact. Major malaria vectors principally bite human hosts around the feet and ankles. Consequently, this study investigated whether sandals treated with efficacious spatial repellents can protect against outdoor biting mosquitoes.
\end{abstract}

Methodology: Sandals affixed with hessian bands measuring $48 \mathrm{~cm}^{2}$ treated with $0.06 \mathrm{~g}, 0.10 \mathrm{~g}$ and $0.15 \mathrm{~g}$ of transfluthrin were tested in large cage semi-field and full field experiments. Sandals affixed with hessian bands measuring $240 \mathrm{~cm}^{2}$ and treated with $0.10 \mathrm{~g}$ and $0.15 \mathrm{~g}$ of transfluthrin were also tested semi field experiments. Human landing catches $(\mathrm{HLC})$ were used to assess reduction in biting exposure by comparing proportions of mosquitoes landing on volunteers wearing treated and untreated sandals. Sandals were tested against insectary reared Anopheles arabiensis mosquitoes in semi-field experiments and against wild mosquito species in rural Tanzania.

Results: In semi-field tests, sandals fitted with hessian bands measuring $48 \mathrm{~cm}^{2}$ and treated with $0.15 \mathrm{~g}, 0.10 \mathrm{~g}$ and $0.06 \mathrm{~g}$ transfluthrin reduced mosquito landings by $45.9 \%$, (95\% confidence interval (C.I.) 28-59\%), 61.1\% (48-71\%), and $25.9 \%$ (9-40\%), respectively compared to untreated sandals. Sandals fitted with hessian bands measuring $240 \mathrm{~cm}^{2}$ and treated with $0.15 \mathrm{~g}$ and $0.10 \mathrm{~g}$ transfluthrin reduced mosquito landings by $59 \%$ (43-71\%) and 64\% (48-74\%), respectively. In field experiments, sandals fitted with hessian bands measuring $48 \mathrm{~cm}^{2}$ and treated with $0.15 \mathrm{~g}$ transfluthrin reduced mosquito landings by $70 \%$ (60-76\%) against Anopheles gambiae sensu lato, and 66.0\% (59-71\%) against all mosquito species combined.

Conclusion: Transfluthrin-treated sandals conferred significant protection against mosquito bites in semi-field and field settings. Further evaluation is recommended for this tool as a potential complementary intervention against malaria. This intervention could be particularly useful for protecting against outdoor exposure to mosquito bites. Additional studies are necessary to optimize treatment techniques and substrates, establish safety profiles and determine epidemiological impact in different settings.

Keywords: Residual malaria transmission, New tools, Transfluthrin, Transfluthrin-treated footwear, Vector borne diseases, Ifakara

\footnotetext{
*Correspondence: psangoro@icipe.org

${ }^{2}$ International Centre for Insect Physiology and Ecology, Nairobi, Kenya

Full list of author information is available at the end of the article
}

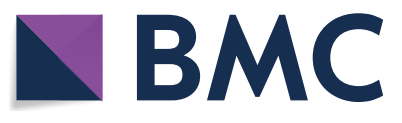

(c) The Author(s) 2020. This article is licensed under a Creative Commons Attribution 4.0 International License, which permits use, sharing, adaptation, distribution and reproduction in any medium or format, as long as you give appropriate credit to the original author(s) and the source, provide a link to the Creative Commons licence, and indicate if changes were made. The images or other third party material in this article are included in the article's Creative Commons licence, unless indicated otherwise in a credit line to the material. If material is not included in the article's Creative Commons licence and your intended use is not permitted by statutory regulation or exceeds the permitted use, you will need to obtain permission directly from the copyright holder. To view a copy of this licence, visit http://creativeco mmons.org/licenses/by/4.0/. The Creative Commons Public Domain Dedication waiver (http://creativecommons.org/publicdomain/ zero/1.0/) applies to the data made available in this article, unless otherwise stated in a credit line to the data. 


\section{Background}

Mosquito control using long-lasting insecticidal nets (LLINs) and indoor residual spraying (IRS) has had a substantial impact on malaria transmission globally [13]. However, deliberate scale up of LLINs and IRS has led to the emergence of behaviourally resilient malaria vectors [4] that evade these tools by increasingly feeding and resting outdoors [5-8]. These changes, associated with the suppression of the once predominant local vector [4-9] attenuate the impact of LLINs and IRS [4]. This shift in mosquito species composition and consequently to mosquito behaviour that define the biological limits of LLINs and IRS, coupled with practices that expose human hosts to outdoor mosquito biting $[10,11]$ have resulted in persistent malaria transmission outdoors (residual transmission) $[12,13]$.

With the increasing significance of outdoor malaria transmission [12], there is need to develop and deploy tools that control mosquito bites outdoors [14]. There are several strategies that are being developed that can be used to tackle transmission outdoors. Killing adult mosquitoes when they feed upon sugar using attractive toxic sugar baits (ATSB) [15-21], when they feed on livestock that have been sprayed with or ingested endectocides [22, 23], use of odour-baited mosquito landing boxes outdoors [24-26], use of topical repellents in the early evenings [27, 28] and larval source management $[29,30]$.

In addition to the above tools, spatial repellents are also being proposed as supplementary to LLINs [31, 32]. Spatial repellents are insecticidal products that act in the vapour phase to prevent human-vector contact by causing mosquitoes to move away from the source of chemical stimulus, interferes with the vector response to stimuli or otherwise causes feeding inhibition [33, 34]. There are several formats through which spatial repellents are dispensed, such as mosquito coils, vaporizer mats and liquid vaporizers $[24,35,36]$. Recently, an alternative emanator delivery format for volatilizing transfluthrin at ambient temperatures have proven efficacious against mosquito bites [37-41]. Although effective, these emanator formats require that the host is confined to a protected air space, therefore, limiting mobility [38-40]. In order to impact outdoor biting, spatial repellents delivery formats must be optimized to protect users wherever they are outdoors [42].

It has previously been reported that the malaria vector, Anopheles gambiae sensu lato (s.l.) prefers to bite humans on the lower limbs and that this behaviour is mediated by convection currents arising off the host $[43,44]$. A recent study demonstrated that the highest densities of bites from these vectors occur on host body parts that are closest to the ground [45], and that protecting these body parts results in significant reduction in mosquito bites to the host $[45,46]$.

Exploiting this mosquito behaviour, this study assessed the impact of integrating spatial repellents into sandals on mosquito bites outdoors. In addition to reducing mosquito bites overall on the human host $[45,46]$, treating footwear with long-lasting spatial repellents also presents an opportunity to overcome concerns of frequent reapplication, which is often encountered when using topical repellents [27], making it prohibitively expensive. Using footwear as a delivery format of spatial repellents will also overcome the challenge of limited mobility of recently developed emanator formats, that require the host to be within the treated air space of stationary emanators [37-39]. Integrating insecticide into footwear that is locally made, low cost and worn ubiquitously across communities on a daily basis will likely promote uptake and the attendant effectiveness as it does not require any change in human behaviour $[27,47,48]$.

\section{Methods \\ Study area and facilities}

Large cage semi-field evaluations were conducted at the Ifakara Health Institute's (IHI) experimental station, in Kining'ina village $\left(8.11^{\circ} \mathrm{S}, 36.67^{\circ} \mathrm{E}\right)$, approximately $6 \mathrm{~km}$ north of Ifakara town, in Kilombero district, south-eastern Tanzania. Experiments were conducted inside two different types of large screened cages. The first was a large multi-compartment system covering $553 \mathrm{~m}^{2}$ in ground area, and $4.5 \mathrm{~m}$ in height (Fig. 1a) [49-51]. The experiments were conducted in two compartments inside this large multi-compartment system each covering $36 \mathrm{~m}^{2}$ in ground area, and $4.5 \mathrm{~m}$ in height. The second cage was a long, tunnel shaped screened system measuring $110 \mathrm{~m}$ long $\times 2 \mathrm{~m}$ wide $\times 2.5 \mathrm{~m}$ high (Fig. 1b, c) [37].

Field experiments were conducted in villages in Kilombero and Ulanga districts, in south-Eastern Tanzania. These study areas experience a wet season between March and June and a dry season in August to October, with a mean annual rainfall of $1200-1400 \mathrm{~mm}$ and a daily temperature range of $20-32{ }^{\circ} \mathrm{C}$ [52]. Malaria is endemic and current transmission is primarily mediated by Anopheles funestus sensu stricto (s.s.) and Anopheles arabiensis. The main malaria control intervention used in the study area is LLINs. Pyrethroid resistance is prevalent in both An. arabiensis [53], and An. funestus [54].

\section{Mosquitoes}

For the semi-field evaluations, laboratory-reared, pyrethroid susceptible $A n$. arabiensis (Ifakara strain) were used in the semi-field experiments. Larvae were fed on Tetramin ${ }^{\circledR}$ fish food and maintained at temperatures of $28-29{ }^{\circ} \mathrm{C}$. Pupae were placed in a separate room in 


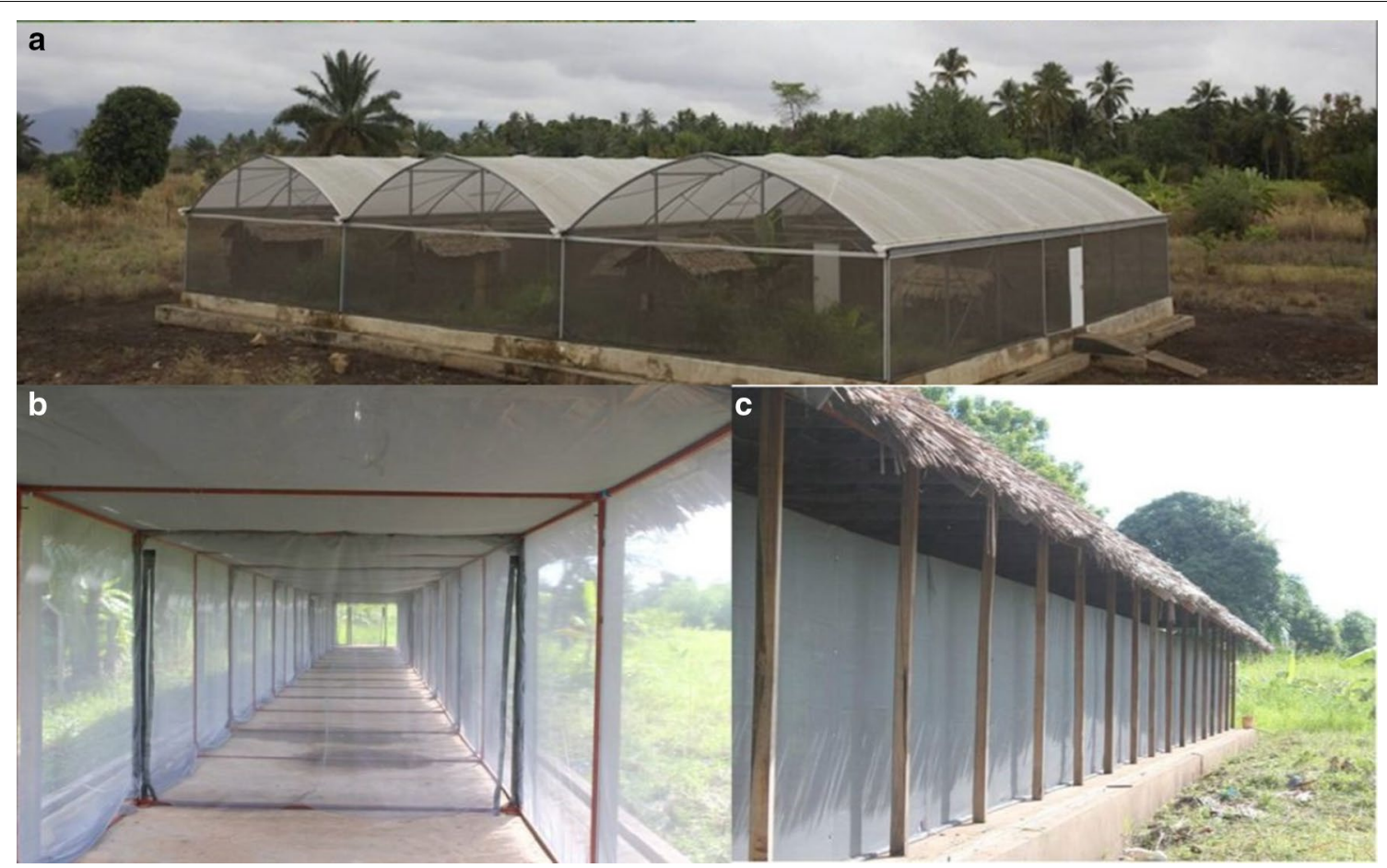

Fig. 1 Pictorial illustration of (IHI) semi field cages, a large multi-compartment system; $\mathbf{b}$ inside sections of the long, tunnel-shaped screened semi-field system; c outside view of the long tunnel-shaped screened semi-field system

emergence bowls inside a $30 \times 30 \times 30 \mathrm{~cm}$ netting cage and a $10 \%$ glucose solution provided for the emergent adults. Temperatures were maintained at $27 \pm 3{ }^{\circ} \mathrm{C}$ and relative humidity at $70-90 \%$. The insectary was maintained at a 12:12 (light:dark) photoperiod. The mosquitoes used in experiments were 4-9 days old nulliparous females. The mosquitoes were starved for $6 \mathrm{~h}$ before each experiment.

\section{Volunteers}

Adult male volunteers (18 to 40 years old) participated in the experiments. The volunteers were trained on objectives, benefits and potential risks of the study and recruited only if they provided written informed consent. All volunteers were highly experienced in the procedure of human landing catch, wherein mosquitoes attempting to bite a volunteer's legs are captured immediately upon landing [55]. The volunteers were instructed not to use any fragranced soap or perfume, tobacco or alcohol throughout the experiment period.

\section{Transfluthrin-treated footwear prototype}

Locally manufactured leather sandals were fitted with transfluthrin-treated hessian fabric affixed onto the straps of the sandals. The bands were fitted on the top leather surface of the sandal straps, so that there was no direct contact with the volunteer's skin (Fig. 2). Hessian fabric was used because it is readily available in Tanzania and has an optimal adsorbent capacity [37]. Transfluthrin,

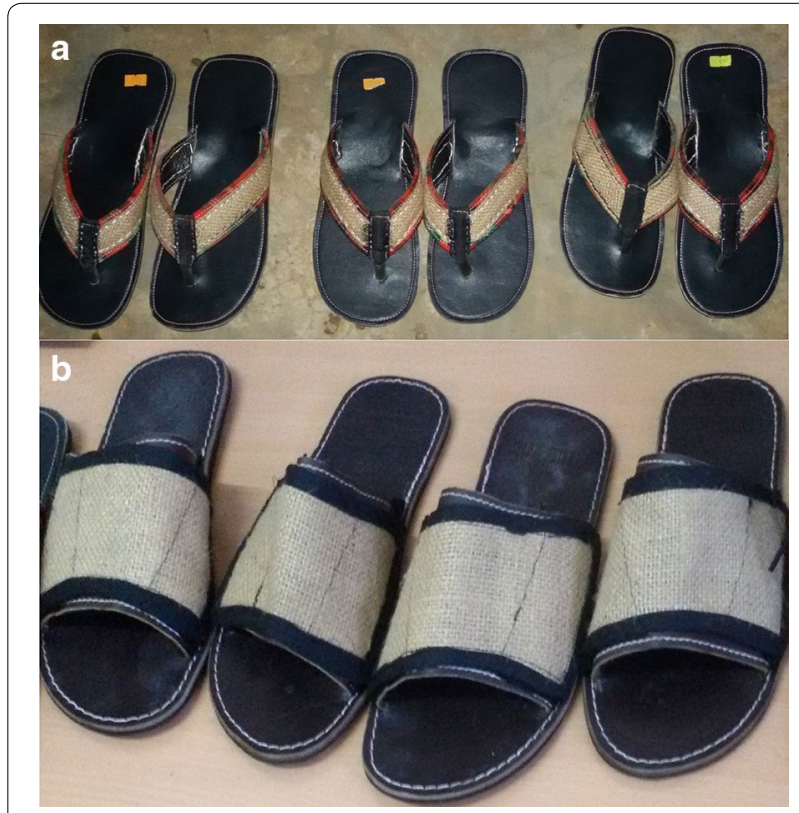

Fig. 2 Prototype designs of the transfluthrin-treated sandals; a design using hessian fabric measuring $240 \mathrm{~cm}^{2}$ and $\mathbf{b}$ design with hessian fabric measuring $48 \mathrm{~cm}^{2}$ 
a pyrethroid insecticide recommended by the World Health Organization (WHO) for control of flying insects, such as mosquitoes and flies [56], was selected as spatial repellent. Transfluthrin is a highly volatile pyrethroid with a vapour pressure of $9 \times 10^{-4} \mathrm{~Pa}$ at $20^{\circ} \mathrm{C}$ [56]. This property makes it suitable for use in tropical regions and at low-cost because no additional heating is required to evaporate the chemical [57]. In addition, previous studies carried out at Ifakara Health Institute have demonstrated efficacy of transfluthrin-impregnated hessian fabric against mosquito bites in semi-field and field experiments [37-40] further affirming the potential of transfluthrintreated hessian substrates in malaria control.

The technical grade transfluthrin used to treat the hessian fabric was donated by SC Johnson (Racine, Wisconsin, USA). Identical sandals treated with axion detergent and water were used as negative controls.

\section{Experiment 1: Semi-field experiments to determine a dose-response relationship for transfluthrin in terms of protection against mosquito bites}

Three different amounts of $97 \%$ technical grade transfluthrin were used to treat the hessian bands attached to the sandals in the first set of experiments; $0.06 \mathrm{~g}, 0.10 \mathrm{~g}$ and $0.15 \mathrm{~g}$ transfluthrin was mixed with $94 \mathrm{ml}, 90 \mathrm{ml}$ and $64 \mathrm{ml}$ of Axion ${ }^{\circledR}$ liquid detergent (Orbit Chemical Industries Ltd, Kenya), respectively, to enable solubility in water [37]. This emulsion was then mixed with $100 \mathrm{ml}$ of water for both $0.06 \mathrm{~g}$ and $0.10 \mathrm{~g}$ transfluthrin and $74 \mathrm{ml}$ of water for $0.15 \mathrm{~g}$ transfluthrin. These amounts were used to ensure there was no left-over emulsion which might undermine the treatment dose.

Rectangular hessian fabric bands measuring $48 \mathrm{~cm}^{2}$ were dipped in the resulting emulsion containing either of the three amounts of transfluthrin and soaked until complete saturation and all the emulsion had been absorbed after which they were suspended at ambient temperatures indoors, protected from direct sun exposure, and allowed to dry for $24 \mathrm{~h}$. Three pairs of sandals were then fitted with the treated hessian fabric bands, each containing either of the three amounts of transfluthrin (Fig. 2b). A pair of matching negative controls for each treatment were prepared in the same way, except the treatments were done with only Axion ${ }^{\circledR}$ liquid detergent and water without any transfluthrin.

Two volunteers were asked to wear knee-length shorts to standardize the area of the lower limbs exposed. They sat on low chairs in experimental compartments measuring $6 \times 6 \mathrm{~m}$ inside the semi-field facility (Fig. 1a). One compartment of the semi-field system was used for testing the treatment while the other tested the control sandal. The experiments were conducted one at a time starting with the sandal fitted with the hessian fabric that had the lowest amount of transfluthrin. Each night, 200 female An. arabiensis mosquitoes were released at the centre of each experimental compartment at 18:00 h, with the volunteer sitting approximately $10 \mathrm{~m}$ from each other. The volunteers collected all mosquitoes attempting to bite the exposed lower limbs for $45 \mathrm{~min}$ of each hour and rested for $15 \mathrm{~min}$. Mosquito collections were done from 18:00 to 06:00 h the next morning. Each volunteer was given a head torch and siphon for aspirating the mosquitoes. The mosquitoes were kept in separate paper cups for each hour of collection. At the end of each night of experiment, the recaptured mosquitoes were killed using petroleum ether, counted and recorded for each hour.

The experiments were conducted in a binary cross-over design, where each amount of transfluthrin (sandal pair) was tested for 8 consecutive nights against the control. Only one sandal pair, containing either of the three treatment amounts was tested per night. On the first night of each experiment testing the different transfluthrin doses, the treatment and control sandals were randomized to the experimental compartments. Volunteers were then rotated each following night between the two experimental compartments of the semi-field system. The experimental compartments were separated by a similar-sized chamber in between that acted as a buffer in the event of spillover effects of the treatment. The treatment and control sandals remained in the same compartment throughout the experiments to minimize the potential impact of residual effect of transfluthrin.

\section{Experiment 2: Semi-field experiments to evaluate the of impact of hessian fabric surface areas on mosquito bites}

Another set of experiments was conducted to assess the impact of surface area of treated hessian fabric on mosquito bites. Hessian fabric pieces measuring $48 \mathrm{~cm}^{2}$ and $240 \mathrm{~cm}^{2}$ were each treated with $0.10 \mathrm{~g}$ and $0.15 \mathrm{~g}$ of transfluthrin using the same methodology described above, affixed onto sandals and their efficacies against mosquito bites evaluated.

The same binary cross-over design as experiment one above was used, and the experiments were replicated 8 times (nights) for each treatment.

\section{Experiment 3: Experimental field evaluation of the efficacy of transfluthrin-treated sandals against bites from wild mosquitoes}

A binary crossover design, similar to the semi-field experiments was used in the two study villages. The $48 \mathrm{~cm}^{2}$ hessian fabric pieces treated with $0.15 \mathrm{~g}$ transfluthrin were evaluated in these experiments as they demonstrated significant efficacy against mosquitoes. The field 
tests were conducted by two volunteers who carried out the experiments over 12 nights in each study village. The tests were conducted outdoors, next to rice fields and away from human dwellings, and these sites, each with two positions, were fixed throughout the experiments. The experiments were conducted on separate nights in the two villages.

The volunteers, wearing transfluthrin-treated sandals sat at a randomly selected position approximately $20 \mathrm{~m}$ away from the volunteer wearing the untreated sandal. The volunteers collected all mosquitoes attempting to bite the exposed lower limbs for $45 \mathrm{~min}$ of each hour and rested for $15 \mathrm{~min}$ from 18:00 to 06:00 h. The volunteers were blinded to the treatment status of the sandals. At the end of the hourly collections, the paper cups holding the mosquitoes were placed in a cool box until the next morning when the mosquitoes were killed using petroleum ether. The mosquitoes in each paper cup were counted by each volunteer and the numbers recorded. The mosquitoes were sorted into anophelines and culicines. Each anopheline was stored in a $5 \mathrm{ml}$ micro-centrifuge tube (Eppendorf ${ }^{\circledR}$ tubes) containing silica gel and later morphologically identified by experienced entomologists. All specimen of An. gambiae s.l. caught were assumed to be An. arabiensis, since contemporaneous molecular analysis of mosquitoes from the same villages have consistently confirmed these to be of An. arabiensis $[28,54,58,59]$.

Other mosquito genera caught were stored in batches of five per micro-centrifuge tube and later identified by experienced entomologists [60].

\section{Experiment 4: Assessment of whether transfluthrin-treated sandals divert host-seeking mosquitoes to persons not wearing the sandals}

This experiment was carried out to determine whether the use of transfluthrin-treated sandals would put nearby non-users at a greater risk of being bitten by diverting mosquitoes to non-users. In this experiment, a volunteer wearing transfluthrin-treated sandals sat at one end of an experimental compartment while another volunteer wearing untreated sandals sat at the other end of the same compartment, approximately $10 \mathrm{~m}$ away. This was the treatment compartment. Four hundred laboratoryreared female $A n$. arabiensis mosquitoes were released from a small cage midway between the two volunteers. The number of mosquitoes attempting to bite the volunteers were caught, throughout the night. Another pair of volunteers sitting in a comparative compartment (control compartment), replicated this experiment using untreated sandals. To establish diversion, the number of mosquitoes caught by the volunteer wearing the untreated sandals in the treatment compartment were compared to the number of the mosquitoes caught by the volunteers in the comparative compartment to determine if the volunteer who sat next to transfluthrin-treated sandals caught more mosquitoes compared to either volunteer in the comparative compartment with untreated sandals only. Similar to the semi-field experiments above, the sandals remained in the same position in each respective chamber and only the volunteers rotated between positions to control for individual attractiveness. The limitations of this design have been outlined in the limitations section of the manuscript.

\section{Experiment 5: Semi-field experiments to assess the efficacy of transfluthrin-treated sandals on laboratory-reared Anopheles and Aedes mosquitoes}

Eight different sandal prototypes (Fig. 3), treated with $0.05 \mathrm{~g}$ of transfluthrin were later developed and tested in the semi field system against the following laboratory reared mosquito species; A (male design 1)-An. arabiensis and Aedes aegypti; B (male design 2)-An. arabiensis; $\mathrm{C}$ (male design 3)-An. arabiensis and Ae. aegypti; D (male design 4)-An. arabiensis; E (female design 1)-An. gambiae s.s. and Ae. aegypti; F (female design 2)-An. arabiensis and Ae. aegypti; G (female design 3)-An. arabiensis and Ae. aegypti and $\mathrm{H}$ (female design 4)-An. arabiensis. All sandals developed could not be tested against all mosquito species because of logistical and cost implications. Different prototype sandals were therefore tested against randomly selected mosquito species. This was done to select the sandal prototype that offered the best protection against the respective randomly selected mosquito species. The results from these experiments were later pooled to evaluate the impact of transfluthrintreated sandals on different mosquito species.

The surface areas of the hessian fabric used for the different sandal designs were as follows; A-395 $\mathrm{cm}^{2}$; $\mathrm{B}-327 \mathrm{~cm}^{2} ; \mathrm{C}-330 \mathrm{~cm}^{2} ; \mathrm{D}-\approx 400 \mathrm{~cm}^{2} ; \mathrm{E}-346 \mathrm{~cm}^{2}$; $\mathrm{F}-780 \mathrm{~cm}^{2} ; \mathrm{G}-640 \mathrm{~cm}^{2}$, and $\mathrm{H}-325 \mathrm{~cm}^{2}$. All the designs developed were treated with $0.05 \mathrm{~g}$ of $97 \%$ technical grade transfluthrin (Shenzhen Sunrising Industry Company, China) following risk assessments of transfluthrin-treated sandals. The treatments followed the same methodology described above.

A binary cross-over design similar to the previous experiments was used to evaluate the impact of the sandals against their respective randomly chosen mosquito species. The experiments were replicated for 6 days for each sandal design. In experiments where Anopheles species were used, experiments were conducted from 18:00 to 00:00 h. (midnight). When Aedes species were used, experiments were conducted from 06:00 to $1200 \mathrm{~h}$. as they are day-biting vectors. 


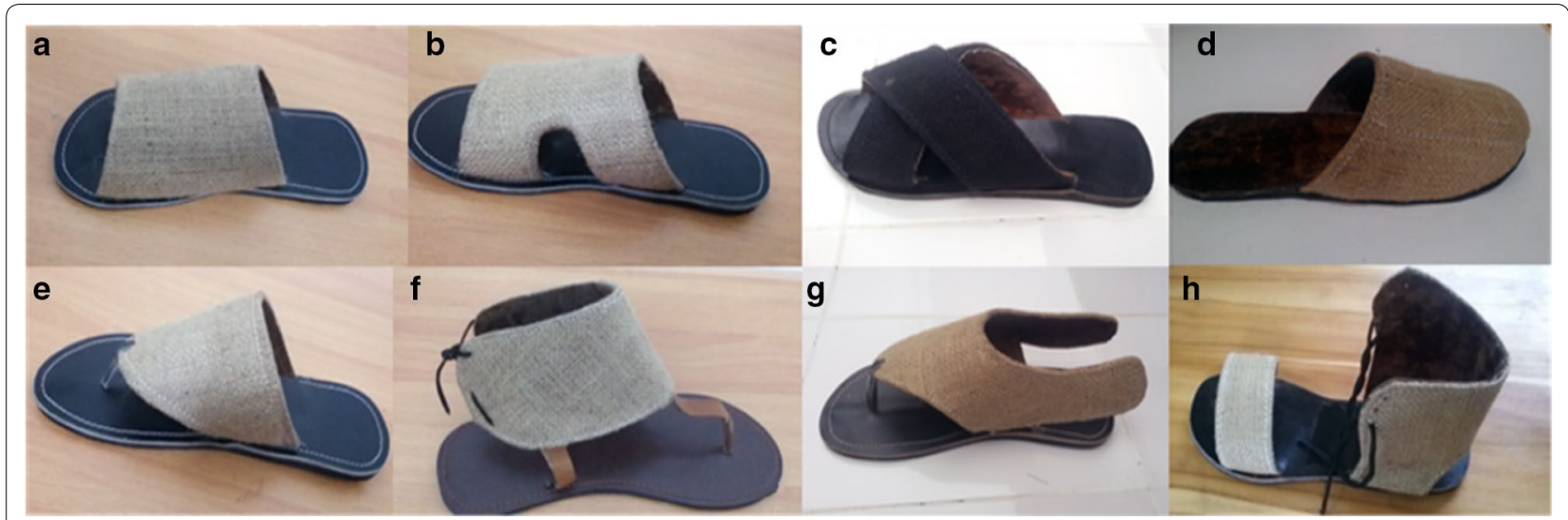

Fig. 3 Prototypes of transfluthrin-treated sandal designs developed; a Male sandal design 1. b Male sandal design 2. c Male sandal design 3. d Male sandal design 4. e Female sandal design 1. f Female sandal design 2. g Female sandal design 3. h Female sandal design 4

One hundred mosquitoes were released in two batches of 50 mosquitoes after every $3 \mathrm{~h}$ in each experimental compartment. The volunteers were asked to wear a customized clothing that covered their whole body but left their lower limbs exposed by pulling the lower parts of their overalls to their knees. Mosquitoes were released into the two experimental compartments at the same time and left to acclimatize for $15 \mathrm{~min}$ before the volunteers entered their respective compartments assigned randomly before the start of the experiments. At the end of the hourly collections, the paper cups holding the mosquitoes were placed in a cool box until the next morning when the mosquitoes were killed using $70 \%$ ethanol. Field technicians counted and the sorted the mosquitoes into anophelines and culicines. Each anopheline was stored in a $5 \mathrm{ml}$ micro-centrifuge tube (Eppendorf tubes) containing silica gel and later taken to the laboratory for further species identification. Other mosquito genera caught were stored in batches of five per micro-centrifuge tube and later identified by experienced entomologists.

\section{Experiment 6: Field experiments to assess the efficacy of sandals treated with transfluthrin on wild mosquito bites}

The field experiments for the two prototype designs of sandals were conducted in Minepa and Lupiro villages in Ulanga district, south-Eastern Tanzania. However, in these experiments, instead of HLCs, the mosquito electrocuting trap (MET) [61, 62] was used to test to the efficacy of transfluthrin-treated sandals against wild mosquitoes.

Similar to the semi-field experiments, a binary cross over design was used when assessing the efficacy of the transfluthrin-treated sandals using the MET in the field. Two METs were placed at least $20 \mathrm{~m}$ apart, next to rice fields and away from human dwellings. The volunteers wearing transfluthrin-treated or untreated sandals were randomly assigned to their positions only on the 1st day of the experiments and on subsequent nights rotated between the two fixed trapping points. The volunteers sat with their lower limbs exposed inside the MET from 18:00 to 00:00 h for the first $45 \mathrm{~min}$ of each hour and collected electrocuted mosquitoes during the last $15 \mathrm{~min}$ of every hour. These experiments were conducted for a total of 12 days, 6 days for each prototype. These two prototypes (male and female sandals design 4) were selected because they provided the best protection against mosquito bites in semi field experiments above.

\section{Statistical analysis}

Data from both the semi-field and field experiments were recorded in a spreadsheet showing date of data collection; name of volunteer; whether the volunteer wore treated or untreated sandals; position of volunteer and the number of mosquitoes caught in each hour.

The effect of transfluthrin-treated sandals on the risk of exposure to mosquito bites was quantified by fitting a generalized linear mixed effects model with a negative binomial distribution to account for the over dispersion of mosquito count data. To account for day to day variation, date was included in the model as a random effect. The treatments on the sandals were included in the model as independent variables and the number of mosquitoes caught of those released as the dependent variable. Variations associated with fluctuations in temperature, humidity, wind direction and speed were assumed to be captured by the date random effect. Date, together with volunteers and hour and were treated as random effects.

To assess whether treated sandals divert mosquito to non-users, the number of mosquitoes caught by the 
untreated sandals in the two experimental compartments were compared. Diversion was modelled using three different values; the untreated sandal that was set up in the same compartment as the treated sandal compared with the two untreated sandals that were used in the comparative compartment. The model was used to derive incidence rate ratios (IRR) for numbers of biting mosquitoes in each of the three scenarios; untreated sandals in the treatment compartment and the two untreated sandals used in the comparative compartment.

\section{Results}

Sandals affixed with hessian fabrics measuring $48 \mathrm{~cm}^{2}$ and treated with $0.15 \mathrm{~g}, 0.10 \mathrm{~g}$ and $0.06 \mathrm{~g}$ transfluthrin reduced mosquito landings by $45.9 \%$, (95\% confidence interval (C.I.) 28-59\%), 61.1\% (48-71\%) and $25.9 \%$ (9-40\%), respectively, when compared to untreated sandals in semi-field experiments (Fig. 4).

In the second set of experiments, hessian fabrics measuring $48 \mathrm{~cm}^{2}$ and $240 \mathrm{~cm}^{2}$ and each treated with both $0.10 \mathrm{~g}$ and $0.15 \mathrm{~g}$ of transfluthrin were compared to determine the impact of surface area on mosquito bites. Sandals affixed with hessian fabric measuring $240 \mathrm{~cm}^{2}$ and treated with $0.15 \mathrm{~g}$ and $0.10 \mathrm{~g}$ transfluthrin reduced mosquito landings by $59 \%(43-71 \%)$ and $64 \%(48-74 \%)$, respectively. Sandals affixed with hessian fabric measuring $48 \mathrm{~cm}^{2}$ hessian fabric and treated with $0.15 \mathrm{~g}$ and $0.10 \mathrm{~g}$ transfluthrin reduced mosquito landings by $57 \%$ (43-67\%) and 44\% (26-56\%), respectively (Fig. 5).

In the third experiment, sandals affixed with hessian fabrics measuring $48 \mathrm{~cm}^{2}$ and treated with $0.15 \mathrm{~g}$ transfluthrin were tested against wild mosquitoes in

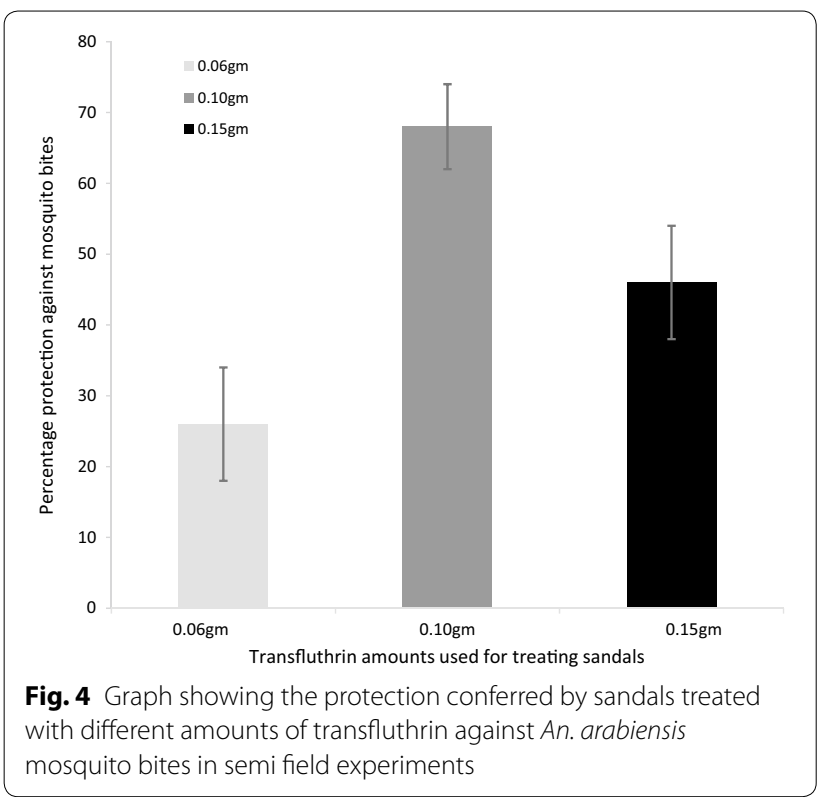

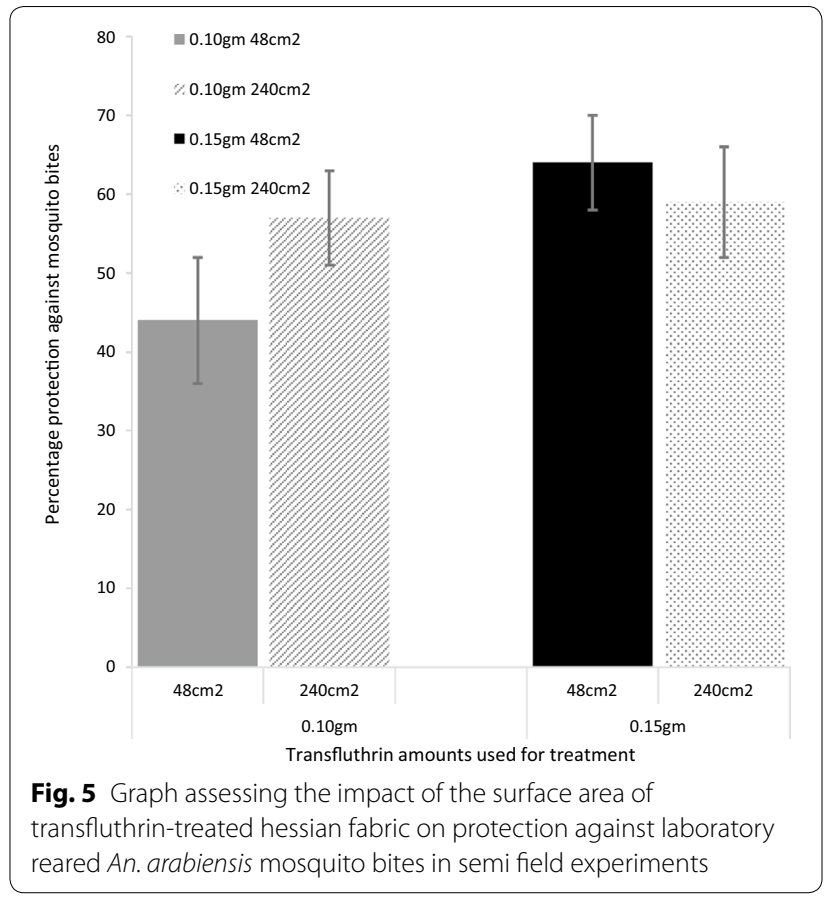

two villages in south-Eastern Tanzania. Transfluthrintreated sandals significantly reduced all mosquito species landing by $65.6 \%$ (95\% C.I. $59-71 \%$ ) compared to the untreated sandals. Against An. gambiae s.l., these sandals reduced mosquito landings by $70.75 \%$ (58.275.7\%) (Table 1).

In the fourth experiment, diversion of transfluthrintreated sandals was assessed. In the experimental compartment the volunteer wearing transfluthrin-treated sandals caught an average of 4.81 (Standard deviation, S.D., 8.32) mosquitoes while the volunteer wearing untreated sandals caught an average of 14.46 (S.D., 24.08) mosquitoes per night. In the comparative compartment, where both volunteers wore untreated sandals, the first volunteer caught an average of 16.25 (S.D., 31.06) mosquitoes while the second volunteer caught an average of 15.34 (S.D., 29.75) mosquitoes per night. Volunteers sitting next to a user of transfluthrin-treated sandal did not receive significantly more mosquito bites when compared to volunteers sitting next to non-users of transfluthrintreated footwear (Table 2).

The fifth experiment evaluated the efficacy of sandals treated with $0.05 \mathrm{~g}$ of transfluthrin against laboratory reared mosquito landings in large cage semi-field experiments. Transfluthrin treated sandals significantly reduced An. arabiensis landings by $55 \%$ (95\% C.I. $48.2-$ $60.9 \%)$ when compared to untreated sandals. When tested against Ae. aegypti, treated sandals reduced mosquito landings by $37.2 \%$ (24.4-47.9\%). Against 
Table 1 Field evaluation of efficacy of transfluthrin-treated sandals against pooled mosquitoes and Anopheles gambiae s.l.

\begin{tabular}{|c|c|c|c|c|c|}
\hline \multirow[t]{2}{*}{ Field experiment } & \multirow[t]{2}{*}{$\begin{array}{l}\text { Incidence rate ratio IRR } \\
\text { (95\% C.I.) }\end{array}$} & \multirow[t]{2}{*}{$p$-value } & \multirow[t]{2}{*}{ z-score } & \multicolumn{2}{|c|}{$\begin{array}{l}\text { Mean number of mosquitoes } \\
\text { (standard deviation) }\end{array}$} \\
\hline & & & & Treatment & Control \\
\hline All mosquito species & $0.34(0.29-0.41)$ & $<0.0001$ & -12.33 & 31.25 (29.64) & $90.64(70.11)$ \\
\hline An. gambiae s.l. & $0.29(0.24-0.40)$ & $<0.0001$ & -7.87 & $1.79(2.73)$ & $5.28(4.99)$ \\
\hline
\end{tabular}

Table 2 Assessment of whether transfluthrin-treated sandals divert host-seeking mosquitoes to persons wearing untreated sandals

\begin{tabular}{|c|c|c|c|c|c|}
\hline \multirow[t]{2}{*}{ Treatment groups } & \multirow[t]{2}{*}{$\begin{array}{l}\text { Incidence rate ratio } \\
\operatorname{IRR}^{\mathrm{a}}(95 \% \text { C.I.) }\end{array}$} & \multirow[t]{2}{*}{ p-value } & \multirow[t]{2}{*}{ z-score } & \multicolumn{2}{|c|}{$\begin{array}{l}\text { Mean number of mosquitoes } \\
\text { (standard deviation) }\end{array}$} \\
\hline & & & & Treatment & Control \\
\hline Repellent-treated sandal (experimental compartment) & $0.32(0.23-0.44)$ & $<0.0001$ & -6.81 & $4.81(8.23)$ & $14.46(24.08)$ \\
\hline Untreated sandal 1 (comparative compartment) & $0.89(0.67-1.18)$ & 0.42 & -0.81 & $15.37(29.75)$ & $14.46(24.08)$ \\
\hline Untreated sandal 2 (comparative compartment) & $0.80(0.59-1.07)$ & 0.13 & -1.53 & $16.25(31.01)$ & $14.46(24.08)$ \\
\hline
\end{tabular}

a The reference sandal was the untreated sandal in the experimental chamber

Table 3 Semi-field experiments to assess the efficacy of sandals treated with $0.05 \mathrm{~g}$ of transfluthrin against different species of laboratory reared mosquito bites

\begin{tabular}{|c|c|c|c|c|c|}
\hline \multirow[t]{2}{*}{ Mosquito species } & \multirow[t]{2}{*}{$\begin{array}{l}\text { Incidence rate ratio IRR } \\
(95 \% \text { C.I.) }\end{array}$} & \multirow[t]{2}{*}{$p$-value } & \multirow[t]{2}{*}{ z-score } & \multicolumn{2}{|c|}{$\begin{array}{l}\text { Mean number of mosquitoes } \\
\text { (standard deviation) }\end{array}$} \\
\hline & & & & Treatment & Control \\
\hline An. arabiensis & $0.45(0.39-0.52)$ & $<0.0001$ & -11.09 & $4.05(3.77)$ & $8.95(6.48)$ \\
\hline Ae. aegypti & $0.62(0.52-0.75)$ & $<0.0001$ & -4.90 & $7.20(5.97)$ & $11.71(10.89)$ \\
\hline An. gambiae s.s. & $0.39(0.30-0.53)$ & $<0.0001$ & -6.46 & $3.94(3.29)$ & $9.56(5.56)$ \\
\hline
\end{tabular}

Table 4 Field experiments to assess the efficacy of sandals treated with $\mathbf{0 . 0 5} \mathbf{g}$ of transfluthrin against different species of wild mosquitoes using the Mosquito electrocuting trap (MET)

\begin{tabular}{|c|c|c|c|c|c|}
\hline \multirow[t]{2}{*}{ Mosquito species } & \multirow[t]{2}{*}{$\begin{array}{l}\text { Incidence rate ratio IRR } \\
(95 \% \text { C.I. })\end{array}$} & \multirow[t]{2}{*}{$p$-value } & \multirow[t]{2}{*}{ z-score } & \multicolumn{2}{|c|}{$\begin{array}{l}\text { Mean number of mosquitoes } \\
\text { (standard deviation) }\end{array}$} \\
\hline & & & & Treatment & Control \\
\hline An. gambiae s.l. & $0.50(0.32-0.79)$ & 0.003 & -2.98 & $3(4.73)$ & $5.63(8.08)$ \\
\hline Culex spp. & $0.59(0.46-0.76)$ & $<0.0001$ & -4.16 & $2.56(3.04)$ & $4.11(3.71)$ \\
\hline
\end{tabular}

An. gambiae s.s. transfluthrin-treated sandals reduced mosquito landings by $60.3 \%$ (47.4-70\%) (Table 3 ).

In the sixth set of experiments, the efficacy of sandals treated with $0.05 \mathrm{~g}$ of transfluthrin was evaluated against wild mosquito species in the field. Transfluthrin-treated sandals reduced An. gambiae s.l. landings by $50 \%$ (95\% C.I. 21.4-68.2\%) and Culex spp. landings by $41 \%(23.8-53.7 \%)$ (Table 4$)$.

\section{Discussion}

Use of transfluthrin-treated sandals reduced exposure to An. gambiae s.l. landings by at least $50 \%$ and $40 \%$ against Culex mosquitoes. Transfluthrin-treated sandals also provided useful protection against Ae. aegypti mosquitoes. This study adds to the mounting body of evidence that these tools (transfluthrin-impregnated substrates) can provide personal protection against different species 
of mosquito species and the growing arsenal of vector control tools that are currently being developed to supplement LLINs and IRS in the context of outdoor mosquito exposure [37-39, 41].

While the increase in transfluthrin amounts used for treatment may appear to be associated with increase in protection, there was no significant difference in reduction of mosquito landings when using either $0.10 \mathrm{~g}$ or $0.15 \mathrm{~g}$ transfluthrin. This suggests that increasing the amounts of spatial repellent used for treatment of sandals above a certain amount may not necessarily provide additional protection against mosquito bites. This finding is consistent with a previous study where $2 \mathrm{ml}$ and $10 \mathrm{ml}$ dose of transfluthrin were found to have no difference in bites reductions [40]. This may imply that like topical repellents, there might be a plateau in the protection provided by higher concentrations of transfluthrin repellents $[41,63]$.This study did not find any impact of treated surface areas on protection efficacy against mosquitoes. However, previous studies using treated hessian ribbons of about $2 \mathrm{~m}$ long provided greater protection efficacy against mosquito bites compared to the findings of this study [38-40]. The lack of impact of surface area of hessian substrate on mosquito landings observed in this study may mean that the differences in the surface areas of the substrates used in this study may not have been large enough to demonstrate an observable effect. These observations indicate that surface area of treated substrates might be an important factor to consider when developing these types of interventions.

Similar to other studies that exploited the potential use of transfluthrin-impregnated substrates as mosquito control tools, this study did not demonstrate any diversion of mosquito bites to non-users of transfluthrin-treated sandals [40]. Consequently, potential employment of transfluthrin-treated sandals is unlikely to place non-users at a greater risk of mosquito bites. This is one advantage that this intervention possesses over topical repellents [64, 65].

In addition to the aspect of diversion, transfluthrintreated sandals also overcome the issue of daily compliance and reapplication that is faced with topical repellents and other spatial emanators, such as mosquito coils, as footwear is something that is already used ubiquitously in the community. This tool will, therefore, not require users to change their behaviour [66], a feature that will facilitate its acceptability and by extension effectiveness [48]. As a result, use of footwear may result in quicker uptake and extensive coverage which will likely make this tool effective in its implementation and scale up. More importantly is that repellent-treated sandals can be used outdoors and during the day where the effect of LLINs and IRS is attenuated [67], offering a complementary tool to tackle this niche of residual transmission and drive towards the goal of malaria elimination.

Despite the potential presented by repellent-treated sandals as a malaria control tool, it should be noted that these results were obtained from a single study. To realize the potential of this tool, there is need to conduct detailed additional studies to optimize the dose and formulations used in treatment of substrates and determine the release rates of the repellents into the airspace. There will also be need for public engagement and education of the efficacy, safe disposal techniques, retreatment and safety profiles of this technology.

Even though experiments to determine the longevity of protection provided by transfluthrin-treated sandals were not carried out, other studies have demonstrated the efficacy of transfluthrin-treated hessian substrates to last longer than 6 months [37-40]. Improved treatment/ impregnation or encapsulation methods of the active ingredient are necessary to ensure safe optimal concentrations. In addition, the optimal substrates and surface areas to be treated/impregnated should be further explored to in order to establish the longevity of protection conferred by this tool.

One limitation of this study was that the treatments were not rotated between the experimental compartments in the semi-field experiments and the impact of the treated sandals may have been as a result of the experimental compartments and not the treatment itself. However, this outcome is unlikely as this bias was controlled for during the field experiments where the treatments were rotated between the experimental positions and the treated sandals still demonstrated significant reduction in mosquito landings.

Another limitation is that this study did not determine the longevity of protection, the area of protection 'bubble' provided and the impact under different environmental and epidemiological backgrounds of transfluthrin-treated sandals.

A third limitation is that mosquitoes were collected from only the lower limbs of the volunteers. It is possible that mosquitoes did bite other parts of the body that were not measured. Further studies need to be conducted to test the impact of the treated sandals on the whole human body.

\section{Conclusion}

Transfluthrin-treated sandal provides a potential tool that could be used to attack residual malaria transmission that is mediated by early and outdoor biting An. arabiensis s.l. However, further studies will be required to optimize this tool before it can be deployed for testing under epidemiological conditions. Cost-benefit analysis as well 
as acceptability studies will also need to be conducted to determine the impact of this tool.

\section{Acknowledgements}

The authors would like to thank the community of Mbingu and Lupiro villages who allowed us to carry out our study in their community. We would like to thank the volunteers who participated in the experiments; Aniceth Kihonda, Saidi Nurdin, Moses Mlagile, Dickson Kihinda, Nasoro llumbo, Ibrahim Mkesa, Boniface Magwila and Nicolaus Mhumbira and Andrew Kafwenji. We would like to thank Prof. Gerry Killeen, Dr. Ulrike Filinger and Dr. David Tchouassi for their inputs to this manuscript. We would also like to thank the USAID for funding implementation of the project and Innovative vector control consortium (IVCC) for their technical support in determining safety profiles. Lastly, we would also like to thank SC Johnson and Sons for donating the repellent used in this study.

\section{Authors' contributions}

OS and TG contributed equally to this study. OS conceived the study and developed the transfluthrin-treated sandal prototype. OS and TG designed and implemented the study. TG conducted the experiments. LF managed the project and supervised the experiments. WM and AL contributed to the study design. EM participated in and supervised the experiments. FO, NG and PC offered oversight and expert advice. OS wrote the first draft of the manuscript with input from TG, FO and PC. All authors revised the manuscript. All authors read and approved the final manuscript.

\section{Funding}

This study was supported by Stars in Global Health; Grand Challenges Canada, Grant number 55-0442-01 which was granted to OS. FOO was also supported by a Wellcome Trust Intermediate Research Fellowship (Grant number: WT102350/Z/13/Z).

\section{Availability of data and materials}

The datasets used and/or analysed during the current study are available from the corresponding author on reasonable request.

\section{Ethics approval and consent to participate}

Ethical approval for the study was obtained from Ifakara Health Institute Institutional Review Board (IHI-IRB) —IHI/IRN/No; 18-2014 and the Tanzanian National Institute of Medical Research (NIMR/HQ/R.8a/NOL IX/2199). All volunteers were asked for verbal as well as written informed consent to participate in the study after study objectives had been explained to them and all their questions answered. Malaria tests were provided to all volunteers before, during and after the study, with the intention that only malaria-free individuals would be allowed to participate in the experiments. All volunteers involved in HLCs in the field were provided with prophylaxis (250 mg Mefloquine taken orally) once every week to prevent malaria infections during the course of the experiments. In addition, treatment (Coartem ${ }^{\circledR}, 80 \mathrm{mg}$ artemether and $480 \mathrm{mg}$ lumefantrine for 3 days) was made available in case any of the participants became ill.The initial experiments reported in this study conducted from January 2014 to October 2015, were conducted using human landing catches (HLC). The subsequent experiments conducted from October 2016 to September 2018 employed the use of the mosquito electrocuting trap (MET) after Ifakara health institute banned the use of HLC in experiments.

\section{Consent for publication}

This manuscript has been approved for publication by National Institute of Medical Research, United Republic of Tanzania, Reference number: NIMR/ HQ/P.12 VOL XXIX/25.

\section{Competing interests}

The authors declare no competing interests.

\section{Author details}

${ }^{1}$ Environmental Health and Ecological Sciences, Ifakara Health Institute, Ifakara, Tanzania. ${ }^{2}$ International Centre for Insect Physiology and Ecology, Nairobi, Kenya. ${ }^{3}$ Institute of Biodiversity, Animal Health and Comparative Medicine, University of Glasgow, Glasgow, UK. ${ }^{4}$ Pan African Mosquito Control Association, Nairobi, Kenya. ${ }^{5}$ School of Public Health, Faculty of Health Sciences, University of the Witwatersrand, Johannesburg, South Africa. ${ }^{6}$ School of Life Sciences and Bio Engineering, The Nelson Mandela, African Institution of Science and Technology, Tengeru, Arusha, United Republic of Tanzania.

Received: 21 January 2020 Accepted: 30 March 2020

Published online: 08 April 2020

\section{References}

1. Bhatt S, Weiss DJ, Cameron E, Bisanzio D, Mappin B, Dalrymple U, et al. The effect of malaria control on Plasmodium falciparum in Africa between 2000 and 2015. Nature. 2015;526:207-11.

2. WHO. World malaria report 2018. Geneva: World Health Organization; 2018. http://www.who.int/publications-detail/world-malaria-report-2018. Accessed 6 Nov 2019.

3. WHO. World malaria report 2019. Geneva: World Health Organization; 2019. http://www.who.int/publications-detail/world-malaria-report-2019. Accessed 11 Dec 2019

4. Govella NJ, Chaki PP, Killeen GF. Entomological surveillance of behavioural resilience and resistance in residual malaria vector populations. Malar J. 2013;12:124.

5. Russell TL, Govella NJ, Azizi S, Drakeley CJ, Kachur SP, Killeen GF. Increased proportions of outdoor feeding among residual malaria vector populations following increased use of insecticide-treated nets in rural Tanzania. Malar J. 2011;10:80.

6. Reddy MR, Overgaard HJ, Abaga S, Reddy VP, Caccone A, Kiszewski AE, et al. Outdoor host seeking behaviour of Anopheles gambiae mosquitoes following initiation of malaria vector control on Bioko Island, Equatorial Guinea. Malar J. 2011;10:184.

7. Moiroux N, Gomez MB, Pennetier C, Elanga E, Djènontin A, Chandre F, et al. Changes in Anopheles funestus biting behavior following universal coverage of long-lasting insecticidal nets in Benin. J Infect Dis. 2012;206:1622-9.

8. Bugoro H, Cooper RD, Butafa C, Iro'ofa C, Mackenzie DO, Chen C-C, et al. Bionomics of the malaria vector Anopheles farauti in Temotu Province, Solomon Islands: issues for malaria elimination. Malar J. 2011;10:133.

9. Bayoh MN, Mathias DK, Odiere MR, Mutuku FM, Kamau L, Gimnig JE, et al. Anopheles gambiae: historical population decline associated with regional distribution of insecticide-treated bed nets in western Nyanza Province, Kenya. Malar J. 2010;9:62.

10. Moshi IR, Ngowo H, Dillip A, Msellemu D, Madumla EP, Okumu FO, et al. Community perceptions on outdoor malaria transmission in Kilombero Valley, Southern Tanzania. Malar J. 2017;16:274.

11. Moshi IR, Manderson L, Ngowo HS, Mlacha YP, Okumu FO, Mnyone LL. Outdoor malaria transmission risks and social life: a qualitative study in South-Eastern Tanzania. Malar J. 2018;17:397.

12. Sherrard-Smith E, Skarp JE, Beale AD, Fornadel C, Norris LC, Moore SJ, et al. Mosquito feeding behavior and how it influences residual malaria transmission across Africa. Proc Natl Acad Sci USA. 2019;116:15086-95.

13. Durnez L, Coosemans M. Residual transmission of malaria: an old issue for new approaches. In: Manguin S, editor. Anopheles mosquitoes-new insights into malaria vectors. Rijeka: InTechOpen; 2013.

14. Zhu L, Müller GC, Marshall JM, Arheart KL, Qualls WA, Hlaing WM, et al. Is outdoor vector control needed for malaria elimination? An individualbased modelling study. Malar J. 2017;16:266

15. Müller GC, Junnila A, Schlein Y. Effective control of adult Culex pipiens by spraying an attractive toxic sugar bait solution in the vegetation near larval habitats. J Med Entomol. 2010;47:63-6.

16. Beier JC, Müller GC, Gu W, Arheart KL, Schlein Y. Attractive toxic sugar bait (ATSB) methods decimate populations of Anopheles malaria vectors in arid environments regardless of the local availability of favoured sugarsource blossoms. Malar J. 2012;11:31.

17. Müller GC, Beier JC, Traore SF, Toure MB, Traore MM, Bah S, et al. Successful field trial of attractive toxic sugar bait (ATSB) plant-spraying methods against malaria vectors in the Anopheles gambiae complex in Mali, West Africa. Malar J. 2010;9:210.

18. Müller GC, Junnila A, Qualls W, Revay EE, Kline DL, Allan S, et al. Control of Culex quinquefasciatus in a storm drain system in Florida using attractive toxic sugar baits. Med Vet Entomol. 2010;24:346-51. 
19. Schlein Y, Müller GC. An approach to mosquito control: using the dominant attraction of flowering Tamarix jordanis trees against Culex pipiens. J Med Entomol. 2008;45:384-90.

20. Müller GC, Schlein Y. Efficacy of toxic sugar baits against adult cisterndwelling Anopheles claviger. Trans R Soc Trop Med Hyg. 2008;102:480-4.

21. Müller G, Schlein Y. Sugar questing mosquitoes in arid areas gather on scarce blossoms that can be used for control. Int J Parasitol. 2006;36:1077-80.

22. Chaccour CJ, Kobylinski KC, Bassat Q, Bousema T, Drakeley C, Alonso P, et al. Ivermectin to reduce malaria transmission: a research agenda for a promising new tool for elimination. Malar J. 2013;12:153.

23. Poché RM, Burruss D, Polyakova L, Poché DM, Garlapati RB. Treatment of livestock with systemic insecticides for control of Anopheles arabiensis in western Kenya. Malar J. 2015;14:351.

24. Andrés M, Lorenz LM, Mbeleya E, Moore SJ. Modified mosquito landing boxes dispensing transfluthrin provide effective protection against Anopheles arabiensis mosquitoes under simulated outdoor conditions in a semi-field system. Malar J. 2015;14:255.

25. Mmbando AS, Okumu FO, Mgando JP, Sumaye RD, Matowo NS, Madumla $E$, et al. Effects of a new outdoor mosquito control device, the mosquito landing box, on densities and survival of the malaria vector, Anopheles arabiensis, inside controlled semi-field settings. Malar J. 2015;14:494.

26. Matowo NS, Koekemoer LL, Moore SJ, Mmbando AS, Mapua SA, Coetzee $M$, et al. Combining synthetic human odours and low-cost electrocuting grids to attract and kill outdoor-biting mosquitoes: field and semi-field evaluation of an improved mosquito landing box. PLOS ONE. 2016;11:e0145653.

27. Wilson AL, Chen-Hussey V, Logan JG, Lindsay SW. Are topical insect repellents effective against malaria in endemic populations? A systematic review and meta-analysis. Malar J. 2014;13:446.

28. Sangoro O, Turner E, Simfukwe E, Miller JE, Moore SJ. A cluster-randomized controlled trial to assess the effectiveness of using 15\% DEET topical repellent with long-lasting insecticidal nets (LLINs) compared to a placebo lotion on malaria transmission. Malar J. 2014;13:324.

29. Fillinger U, Lindsay SW. Larval source management for malaria control in Africa: myths and reality. Malar J. 2011;10:353.

30. Tusting LS, Thwing J, Sinclair D, Fillinger U, Gimnig J, Bonner KE, et al. Mosquito larval source management for controlling malaria. Cochrane Database Syst Rev. 2013;8:CD008923.

31. Achee N, Masuoka P, Smith P, Martin N, Chareonviryiphap T, Polsomboon $S$, et al. Identifying the effective concentration for spatial repellency of the dengue vector Aedes aegypti. Parasit Vectors. 2012;5:300.

32. WHO. Guidelines for malaria vector control. Geneva: World Health Organization. https://apps.who.int/iris/bitstream/handle/10665/310862/97892 41550499-eng.pdf?ua=1. Accessed 11 June 2019.

33. WHO Pesticide Evaluation Scheme (WHOPES). Guidelines for efficacy testing of spatial repellents. Geneva: World Health Organization; 2013. http://apps.who.int/iris/bitstream/10665/78142/1/9789241505024_eng pdf. Accessed 19 Oct 2017.

34. Achee NL, Bangs MJ, Farlow R, Killeen GF, Lindsay S, Logan JG, et al. Spatial repellents: from discovery and development to evidence-based validation. Malar J. 2012;11:164.

35. WHO Pesticide Evaluation Scheme (WHOPES). Guidelines for efficacy testing of household insecticide products. Geneva: World Health Organization; 2009. https://apps.who.int/iris/bitstream/handle/10665/70071/ WHO_HTM_NTD_WHOPES_2009.3_eng.pdf?sequence=1. Accessed 11 June 2019.

36. Ogoma SB, Moore SJ, Maia MF. A systematic review of mosquito coils and passive emanators: defining recommendations for spatial repellency testing methodologies. Parasit Vectors. 2012;5:287.

37. Ogoma SB, Ngonyani H, Simfukwe ET, Mseka A, Moore J, Killeen GF. Spatial repellency of transfluthrin-treated hessian strips against laboratoryreared Anopheles arabiensis mosquitoes in a semi-field tunnel cage. Parasit Vectors. 2012;5:54.

38. Masalu JP, Finda M, Okumu FO, Minja EG, Mmbando AS, Sikulu-Lord MT, et al. Efficacy and user acceptability of transfluthrin-treated sisal and hessian decorations for protecting against mosquito bites in outdoor bars. Parasit Vectors. 2017;10:197.

39. Govella N, Ogoma S, Paliga J, Chaki P, Killeen G. Impregnating hessian strips with the volatile pyrethroid transfluthrin prevents outdoor exposure to vectors of malaria and lymphatic filariasis in urban Dar es Salaam, Tanzania. Parasit Vectors. 2015;8:322.

40. Ogoma SB, Mmando AS, Swai JK, Horstmann S, Malone D, Killeen GF. A low technology emanator treated with the volatile pyrethroid transfluthrin confers long term protection against outdoor biting vectors of lymphatic filariasis, arboviruses and malaria. PLoS Negl Trop Dis. 2017;11:e0005455.

41. Ogoma SB, Ngonyani H, Simfukwe ET, Mseka A, Moore J, Maia MF, et al. The mode of action of spatial repellents and their impact on vectorial capacity of Anopheles gambiae sensu stricto. PLoS ONE. 2014;9:e110433.

42. Killeen GF, Moore SJ. Target product profiles for protecting against outdoor malaria transmission. Malar J. 2012;11:17.

43. Dekker T, Takken W, Knols BGJ, Bouman E, Laak S, Bever A, et al. Selection of biting sites on a human host by Anopheles gambiae s.S., An. arabiensis and An. quadriannulatus. Entomol Exp Appl. 1998:87:295-300.

44. De Jong R, Knols BGJ. Selection of biting sites on man by two malaria mosquito species. Experientia. 1995;51:80-4.

45. Braack L, Hunt R, Koekemoer LL, Gericke A, Munhenga G, Haddow AD, et al. Biting behaviour of African malaria vectors: 1. Where do the main vector species bite on the human body? Parasit Vectors. 2015;8:76.

46. Durrheim DN, Govere JM. Malaria outbreak control in an African village by community application of "deet" mosquito repellent to ankles and feet. Med Vet Entomol. 2002;16:112-5.

47. Gryseels C, Uk S, Sluydts V, Durnez L, Phoeuk P, Suon S, et al. Factors influencing the use of topical repellents: implications for the effectiveness of malaria elimination strategies. Sci Rep. 2015;5:16847.

48. Kiszewski AE, Darling ST. Estimating a mosquito repellent's potential to reduce malaria in communities. J Vector Borne Dis. 2010;47:217-21.

49. Lwetoijera D, Harris C, Kiware S, Dongus S, Devine GJ, McCall PJ, et al. Effective autodissemination of pyriproxyfen to breeding sites by the exophilic malaria vector Anopheles arabiensis in semi-field settings in Tanzania. Malar J. 2014;13:161.

50. Ng'habi KR, Mwasheshi D, Knols BG, Ferguson HM. Establishment of a self-propagating population of the African malaria vector Anopheles arabiensis under semi-field conditions. Malar J. 2010;9:356.

51. Ferguson HM, Ng'habi KR, Walder T, Kadungula D, Moore SJ, Lyimo I, et al. Establishment of a large semi-field system for experimental study of African malaria vector ecology and control in Tanzania. Malar J. 2008;7:158.

52. Koutsouris AJ, Chen D, Lyon SW. Comparing global precipitation data sets in eastern Africa: a case study of Kilombero Valley, Tanzania. Int J Climatol. 2016;36:2000-14

53. Matowo NS, Munhenga G, Tanner M, Coetzee M, Feringa WF, Ngowo $\mathrm{HS}$, et al. Fine-scale spatial and temporal heterogeneities in insecticide resistance profiles of the malaria vector, Anopheles arabiensis in rural south-eastern Tanzania. Wellcome Open Res. 2017;2:96.

54. Kaindoa EW, Matowo NS, Ngowo HS, Mkandawile G, Mmbando A, Finda $\mathrm{M}$, et al. Interventions that effectively target Anopheles funestus mosquitoes could significantly improve control of persistent malaria transmission in south-eastern Tanzania. PLoS ONE. 2017;12:e0177807.

55. WHO. Manual on practical entomology in malaria. Geneva: World Health Organization; 1975

56. WHO Pesticide Evaluation Scheme (WHOPES). WHO specifications and evaluations for public health pesticides transfluthrin. Geneva: World Health Organization; 2016. http://www.who.int/whopes/quality/Trans fluthrin_eval_specs_WHO_May_2016.pdf?ua=1. Accessed 19 Oct 2017.

57. Kawada H, Maekawa Y, Tsuda Y, Takagi M. Laboratory and field evaluation of spatial repellency with metofluthrin-impregnated paper strip against mosquitoes in Lombok Island, Indonesia. J Am Mosq Control Assoc. 2004;20:292-8.

58. Ngowo HS, Kaindoa EW, Matthiopoulos J, Ferguson HM, Okumu FO. Variations in household microclimate affect outdoor-biting behaviour of malaria vectors. Wellcome open Res. 2017;2:102.

59. Maia MF, Kreppel K, Mbeyela E, Roman D, Mayagaya V, Lobo NF, et al. A crossover study to evaluate the diversion of malaria vectors in a community with incomplete coverage of spatial repellents in the Kilombero Valley, Tanzania. Parasit Vectors. 2016;9:451.

60. Edwards F. Mosquitoes of the Ethiopian region. British Museum (Natural History); 1941. http://www.worldcat.org/title/mosquitoes-of-the-ethio pian-region-vol-3-h-culicine-adults-and-pupae/oclc/988628093?refer er=di\&ht=edition. Accessed 23 Oct 2017.

61. Meza FC, Kreppel KS, Maliti DF, Mlwale AT, Mirzai N, Killeen GF, et al. Mosquito electrocuting traps for directly measuring biting rates and 
host-preferences of Anopheles arabiensis and Anopheles funestus outdoors. Malar J. 2019;18:83.

62. Govella NJ, Maliti DF, Mlwale AT, Masallu JP, Mirzai N, Johnson PCD, et al. An improved mosquito electrocuting trap that safely reproduces epidemiologically relevant metrics of mosquito human-feeding behaviours as determined by human landing catch. Malar J. 2016;15:465.

63. Fradin MS, Day JF. Comparative efficacy of insect repellents against mosquito bites. N Engl J Med. 2002;347:13-8

64. Maia MF, Onyango SP, Thele M, Simfukwe ET, Turner EL, Moore SJ. Do topical repellents divert mosquitoes within a community? - Health equity implications of topical repellents as a mosquito bite prevention tool. PLOS ONE. 2013;8:e84875.
65. Maia M, Sangoro P, Thele M, Turner E, Moore S. Do topical repellents divert mosquitoes within a community? Malar J. 2012;11:P120.

66. Panter-Brick C, Clarke SE, Lomas H, Pinder M, Lindsay SW. Culturally compelling strategies for behaviour change: a social ecology model and case study in malaria prevention. Soc Sci Med. 2005;62(11):2810-25.

67. Killeen GF. Characterizing, controlling and eliminating residual malaria transmission. Malar J. 2014;13:330

\section{Publisher's Note}

Springer Nature remains neutral with regard to jurisdictional claims in published maps and institutional affiliations.
Ready to submit your research? Choose BMC and benefit from:

- fast, convenient online submission

- thorough peer review by experienced researchers in your field

- rapid publication on acceptance

- support for research data, including large and complex data types

- gold Open Access which fosters wider collaboration and increased citations

- maximum visibility for your research: over $100 \mathrm{M}$ website views per year

At BMC, research is always in progress.

Learn more biomedcentral.com/submissions 